\title{
Heterogeneidad lateral en las muestras de roedores del tramo T1 del Mioceno Medio de Somosaguas (Pozuelo de Alarcón, Madrid)
}

\section{Lateral heterogeneity in rodent samples of the T1 level from the Middle Miocene of Somosaguas (Pozuelo de Alarcón, Madrid)}

\author{
F. Blanco ${ }^{1}$, M. Hernández Fernández $z^{1,2}$ \\ 1 Departamento de Paleontología, Facultad de Ciencias Geológicas, Universidad Complutense de Madrid, José Antonio \\ Novais 2, Madrid 28040, Spain. Email: fblancosegovia@gmail.com \\ 2 Departamento de Geología Sedimentaria y Cambio Medioambiental, Instituto de Geociencias (CSIC, UCM), José Antonio \\ Novais 2, Madrid 28040, Spain.
}

\section{RESUMEN}

Las asociaciones de roedores son utilizadas de manera generalizada en paleontología para realizar inferencias paleoambientales. En ocasiones, asumiendo una homogeneidad lateral en el contenido fosilífero de los niveles estratigráficos, estas inferencias se llevan a cabo utilizando las abundancias relativas de las distintas especies que componen esas asociaciones. No obstante, nunca se ha comprobado experimentalmente dicha homogeneidad espacial en las proporciones específicas dentro de un mismo nivel fosilífero. Para comprobar esta homogeneidad lateral, en este trabajo se compararon dos muestras procedentes del tramo T1 (SomosaguasSur y Cata-3) del yacimiento paleontológico de Somosaguas (Mioceno Medio), correspondientes a dos puntos de muestreo separados unos $30 \mathrm{~m}$. Aunque se mantuvo una composición faunística idéntica desde un punto de vista cualitativo, los análisis realizados por medio de aleatorizaciones de Monte Carlo, indicaron la existencia de diferencias significativas en las abundancias relativas de las distintas especies entre las dos muestras. Estos resultados podrían deberse a 1) un diferente comportamiento sedimentario de las piezas dentales de las distintas especies debido a diferencias en su tamaño; 2) el reducido tamaño de las muestras de sedimento en combinación con la elevada densidad de restos fósiles en el tramo T1 de Somosaguas; 3) una mezcla temporal diferencial relacionada con la presencia de varios abanicos aluviales sucesivos, sumado a cambios poblacionales de la comunidad local de roedores influidos por cambios ambientales durante el Aragoniense. Nuestros resultados muestran la necesidad de ser cautelosos a la hora de realizar inferencias palecológicas basadas en abundancias relativas de especies de micromamíferos.

Palabras clave: Aragoniense; Paleoecología; Técnicas de análisis; Sedimentología; Tafonomía; Muestreo.

\section{ABSTRACT}

Rodent associations are habitually used in palaeoenviromental inferences. Assuming lateral homogeneity in fossil content within stratigraphic levels, sometimes these inferences are performed using the relative abundances of species included in such associations. Nevertheless, this homogeneity has never been checked empirically in

Recibido el 8 de septiembre de 2015 / Aceptado el 13 de abril de 2016 / Publicado online el 23 de noviembre de 2016

Citation / Cómo citar este artículo: F. Blanco \& M. Hernández Fernández (2016). Heterogeneidad lateral en las muestras de roedores del tramo T1 del Mioceno Medio de Somosaguas (Pozuelo de Alarcón, Madrid). Estudios Geológicos 72(2): e052. http://dx.doi. org/10.3989/egeol.42255.386.

Copyright: () 2016 CSIC. This is an open-access article distributed under the terms of the Creative Commons Attribution-Non Commercial (by-nc) Spain 3.0 License. 


\begin{abstract}
species proportions inside the same fossiliferous level. Because of that, in this work we compared two samples from T1 level of the Somosaguas fossil site (middle Miocene), which were separated laterally by $30 \mathrm{~m}$. Although both samples show identical faunal composition from a qualitative viewpoint, analysis realized through Monte Carlo randomizations indicated the existence of significant differences in relative abundances of different species between the two samples. These results could be due to 1) different sedimentary behaviour of dental pieces from different species due to differences in size; 2) small size of sediment samples combined with high density of fossil remains in level T1 of Somosaguas; 3) differential time averaging related to presence of successive alluvial fans, associated to population changes due to environmental changes during the Aragonian. Our results show the need of cautiousness when palaeoenviromental inferences are based on relative abundances of micromammalian species.
\end{abstract}

Keywords: Aragonian; Paleoecology; Analysis techniques; Sedimentology; Taphonomy; Sampling.

\section{Introducción}

Debido a sus características biológicas, los roedores poseen en general ciclos vitales cortos, con estrategias reproductivas de tipo $\mathrm{r}$, lo que les hace adaptarse de manera muy rápida a los cambios ambientales, dejando en el registro fósil evidencias de los mismos. A ello se suma el hecho de que las piezas dentales de estos mamíferos se conservan en gran abundancia en los yacimientos. Como consecuencia las asociaciones de roedores han sido tradicionalmente usadas en paleontología como indicadores paleoambientales (Daams \& Meulen, 1984; Sesé, 1991, 2011; Meulen \& Daams, 1992; Voglino \& Pardiñas, 2005; Hernández Fernández, 2006).

Las abundancias relativas de las diferentes especies constituyentes de las asociaciones de roedores registradas en los yacimientos se han usado para realizar inferencias paleocológicas desde hace décadas (Weerd \& Daams, 1978; Sesé, 1991; Meulen \& Daams, 1992; Dam, 1997; Daams et al., 1999; Cuenca-Bescós et al., 2009; Anemone et al., 2012; Rodrigues et al., 2012; López-García et al., 2013; Fernández García, 2014; Lyman, 2014). Al hacer estas inferencias, se asume de manera implícita que hay una homogeneidad en la composición faunística dentro de un mismo nivel fosilífero. Esta asunción se basaría en la dinámica deposicional de los procesos implicados en la formación de los yacimientos, por la cual habitualmente los restos que conformarían el nivel fosilífero serían resedimentados, en especial en aquellos yacimientos de origen fluvio-lacustre. De este modo las posibles diferencias en cuanto a la distribución espacial de los restos pertenecientes a las distintas especies sería anulada debido a la homogenización producida por resedimentación (Polonio \& López Martínez, 2000; Hernández Fernández et al., 2006; Sainz de los Terreros \& Gómez
Castanedo, 2011). No obstante, no tenemos noticias de que esta homogeneidad composicional asumida se haya comprobado experimentalmente hasta la fecha.

Con el fin de comprobar experimentalmente esta hipotética homogeneidad lateral dentro de un nivel fosilífero, el presente estudio comparó cuantitativamente dos muestras de roedores pertenecientes al mismo estrato fosilífero de un yacimiento de origen fluvio-lacustre. Para ello, hemos elegido el nivel T1 del yacimiento mioceno de Somosaguas, cuya abundancia de restos de micromamíferos y amplia extensión lateral (López-Martínez et al., 2000; Hernández Fernández et al., 2006; Díez-Canseco et al., 2012) hacen de él una zona de muestreo idónea para este fin (Fig. 1). Además, en este yacimiento, Luis (2002) realizó un estudio preliminar mediante métodos de rarefacción, en el cual detectó indicios de una posible heterogeneidad a nivel cualitativo entre Somosaguas 4 y Somosaguas 5 , pertenecientes al mismo tramo fosilífero, basada en la presencia de un ejemplar de una especie única que hace que Somosaguas 5 tenga mayor riqueza específica causando diferencias estadísticas entre ambas muestras.

\section{Material y métodos}

\section{Área de estudio y muestreo}

Elárea de estudio objeto del presente trabajo se sitúa en el municipio madrileño de Pozuelo de Alarcón, en el Campus de Somosaguas de la Universidad Complutense de Madrid. El Área Paleontológica de Somosaguas es una de las más occidentales de la cuenca de Madrid (López-Martínez et al., 2000) y tiene una gran extensión geográfica $\left(>25.000 \mathrm{~m}^{2}\right)$, en la cual se sitúan varios puntos de muestreo diferenciados. Hasta la fecha se han identificado 24 especies de mamíferos presentes en la zona, abarcando 


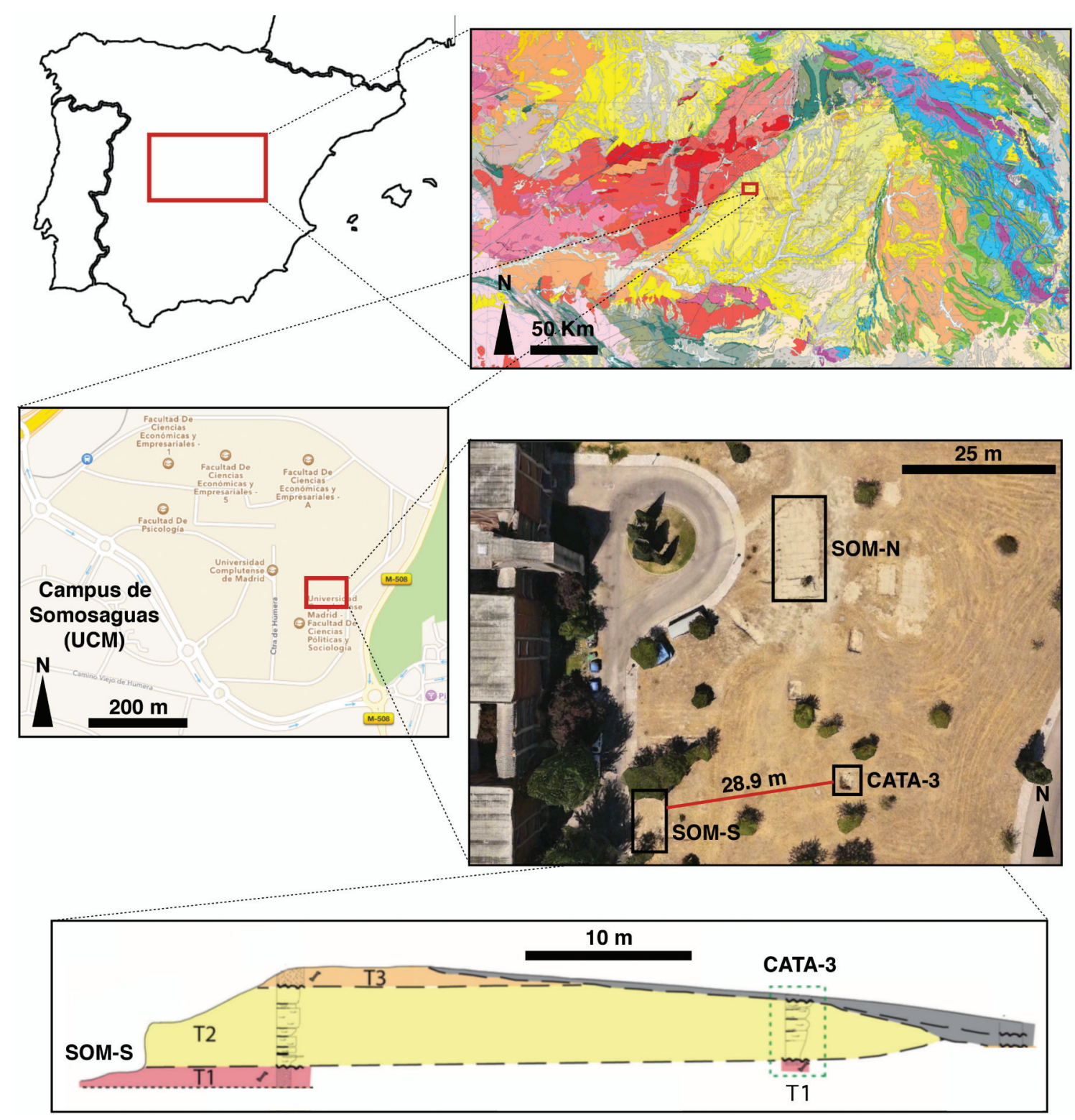

Fig.1.-Localización geográfica y contexto geológico del yacimiento paleontológico de Somosaguas (modificado de Rodríguez Fernández et al., 2016). En las imágenes inferiores se destaca la posición relativa de los dos puntos de muestreo comparados (Cata-3 y Som-S), así como la sección estratigráfica entre los mismos (modificado de Díez-Canseco et al., 2012).

un amplio abanico de tamaños corporales (Perales et al., 2009). En cuanto a los roedores presentes en el yacimiento, Luis \& Hernando (2000) describieron seis especies en Somosaguas-Sur: Heteroxerus grivensis (Forsyth Major, 1893), Microdyromys koegniswaldi de Bruijn, 1966, Microdyromys monspeliensis Aguilar 1977, Armantomys tricristatus López Martínez, 1977, Megacricetodon collongensis
(Mein, 1958) y Fahlbuschia darocensis (Freudenthal, 1963). Meulen et al. (2003) asignaron los restos de Fahlbuschia darocensis a la especie Democricetodon larteti (Schaub, 1925). A estas especies ha de añadirse Cricetodon soriae López-Martínez et al., 2006 in Hernández Fernández et al., 2006, que aparece en Somosaguas-Norte y Somosaguas-5 (Hernández Fernández et al., 2006). Este conjunto faunístico ha 
permitido datar el yacimiento como perteneciente a la Biozona E, dentro de la MN5 (Aragoniense Medio), con una edad aproximada de 14 millones de años (Luis \& Hernando, 2000; Hernández Fernández et al., 2006; Hernández-Ballarín et al., 2011).

Los materiales aflorantes presentes en la zona son arenas y microconglomerados arcósicos con contenido arcilloso variable, que se corresponden con zonas palustres-lacustres y depósitos de abanicos aluviales procedentes del Sistema Central (Mínguez Gandú, 2000; Cuevas-González, 2005; Fesharaki, 2005; Díez-Canseco et al., 2012). Estos sedimentos quedan distribuidos en tres tramos estratigráficos superpuestos, denominados T1, T2 y T3. Mientras que el tramo $\mathrm{T} 2$ es estéril en cuanto a presencia fosilífera (Díez-Canseco, 2011), los tramos T1 y T3 presentan abundantes restos de vertebrados (López Martínez et al., 2000; Hernández Fernández et al., 2006). Concretamente, el tramo T1 muestra una elevada densidad de restos de microvertebrados (Luis \& Hernando, 2000), siendo el tramo de procedencia de las muestras estudiadas en este trabajo.

Para este estudio de la variabilidad lateral en las muestras de micromamíferos del tramo T1, durante la campaña de excavación de Mayo de 2008 se extrajeron aproximadamente $25 \mathrm{~kg}$ de sedimento procedente del fondo de la Cata-3 del yacimiento de Somosaguas (Fig. 1), situada a menos de $30 \mathrm{~m}$ de Somosaguas-Sur, punto de muestreo de Luis \& Hernando (2000). La muestra fue tomada de la parte superior del tramo T1 (entre el techo del nivel y $20 \mathrm{~cm}$ por debajo del mismo), donde se encuentra la mayor concentración de restos fósiles de este nivel, en una posición equivalente en la vertical a la muestreada por Luis \& Hernando (2000) en Somosaguas-Sur.

El sedimento fue secado y posteriormente tratado con la técnica conocida como lavado-tamizado, que consiste en lavar con agua a presión a la vez que se tamiza la muestra (Daams \& Freudenthal, 1988). Con el fin de obtener unos resultados comparables, usamos la misma luz de malla mínima $(0,7 \mathrm{~mm})$ que Luis \& Hernando (2000). Aunque es posible que se produzca una cierta pérdida de restos incompletos, al ser de menor tamaño que esta luz de malla, la mayoría de ellos no serían significativos según el protocolo de análisis de la muestra utilizado (ver más adelante).

$\mathrm{Al}$ final del proceso obtuvimos $791 \mathrm{~g}$ de concentrado que fueron sometidos a triado (picking) de los restos fósiles. Una vez finalizado el proceso se obtuvieron un total de 6.492 restos fósiles, de los cuales 563 correspondieron a piezas dentales. En la colección procedente de Somosaguas-Sur, Luis \& Hernando (2000) muestrearon $50 \mathrm{~kg}$ de sedimento, del cual obtuvieron $3,5 \mathrm{~kg}$ de concentrado, consiguiendo 680 piezas dentales identificables (los fragmentos no identificables no fueron incluidos en la colección).

\section{Análisis de la muestra}

Se identificaron a nivel específico todos los restos dentales identificables encontrados en la Cata-3. A su vez, para poder comparar esta nueva muestra con la de Somosaguas-Sur estudiada por Luis \& Hernando (2000), se examinó toda la colección original, revisando sus asignaciones sistemáticas. Esto permitió separar Microdyromys monspeliensis y Microdyromys koegniswaldi, que estaban identificados de manera conjunta en Luis \& Hernando (2000). Del mismo modo, se registró en Somosaguas-Sur la presencia de una especie no identificada previamente por estos autores, Heteroxerus rubricati Crusafont et al. (1955).

De todos los dientes obtenidos, siguiendo a Weerd \& Daams (1978), para el estudio cuantitativo de la muestra sólo utilizamos los primeros y segundos molares inferiores y superiores $(\mathrm{m} 1+\mathrm{m} 2+\mathrm{M} 1+\mathrm{M} 2)$ presentes en la muestra. Se contabilizaron todos aquellos molares que estaban enteros o eran fragmentos identificables significativos, entendiendo estos como los que representan más de la mitad del diente (Dam, 1997). Se procedió así porque estas piezas dentales están presentes en todas las especies de roedores, independientemente de las distintas fórmulas dentales que puedan presentar, lo que nos sirve para homogeneizar la muestra. Otro factor a tener en cuenta es que estas piezas son, generalmente, los dientes más diagnósticos para la identificación de las especies de roedores. Por último, la abundancia de los terceros molares en las especies de menor tamaño puede verse sesgada debido a que una buena parte de los mismos puede atravesar la luz de malla más pequeña utilizada $(0,7 \mathrm{~mm})$, descartándose su uso para nuestros análisis (Menéndez et al., 2015). Como resultado de este análisis cuantitativo, se han contabilizado 165 primeros y segundos molares de roedores en la Cata-3 y 455 en Somosaguas-Sur. 
Los datos correspondientes a las abundancias de las distintas especies muestreadas procedentes de la Cata-3 se han comparado con las obtenidas en Somosaguas-Sur por medio de dos aproximaciones diferentes.

En primer lugar, debido a que los datos procedentes de la Cata-3 tenían un menor tamaño muestral que los procedentes de Somosaguas-Sur, se procedió a la rarefacción aleatoria en esta última muestra mediante un análisis de Monte Carlo (Manly, 1997). Con ello, se pretendía simular las abundancias relativas de cada especie si el tamaño muestral en Somosaguas-Sur hubiese sido igual al de la muestra de menor tamaño (Cata-3). Por medio del software Microsoft Excel se realizaron 1.000 aleatorizaciones sobre la muestra de Somosaguas-Sur, simulando un tamaño muestral de 165 restos dentales (el tamaño muestral de la Cata-3) mediante la eliminación aleatoria de 290 restos de la muestra. Así se obtuvieron distribuciones nulas para las estimas de frecuencia de cada especie en Somosaguas-Sur, bajo unas condiciones de esfuerzo de muestreo comparables a las de la Cata-3. La hipótesis nula asume que las diferencias de frecuencia para cada especie observadas entre la Cata-3 y Somosaguas-Sur se deberían al azar. La probabilidad ( $p$-valor) de que haya diferencias estadísticamente significativas entre el valor observado en la muestra de la Cata-3 y las muestras aleatorizadas de Somosaguas-Sur se obtuvo a partir de la distribución de valores en estas (prueba de dos colas), por comparación con las proporciones observadas en la Cata-3 para cada una de las especies.

En segundo lugar, con el objetivo de que los análisis también tuviesen en cuenta la variabilidad propia de muestreos aleatorios en la Cata-3, se realizaron dos análisis de Monte Carlo adicionales, tanto sobre la muestra de la Cata-3 como sobre la de Somosaguas-Sur. En este caso, se simularon 1.000 muestras aleatorias con un esfuerzo de muestreo de 100 molares inferiores y superiores en cada una. Este tamaño muestral es habitualmente considerado suficiente en los estudios paleoecológicos para que la muestra se considere representativa de la paleocomunidad original (Weerd \& Daams, 1978; Daams et al., 1999; Casanovas-Vilar \& Agustí, 2007; Hernández Fernández et al., 2007; Gómez Cano et al., 2014). Con los datos obtenidos del análisis Monte Carlo para ambas muestras realizamos un análisis no paramétrico de U de Mann-Whitney (Kayusa, 2001), por medio del software PAST (Hammer et al., 2001) con el fin de calcular si había diferencias significativas entre las frecuencias en ambas muestras para cada una de las especies (Tabla 1 y 2).

\section{Resultados}

Tras el estudio de los restos fósiles procedentes de la Cata-3, se identificaron 7 especies de roedores: Heteroxerus grivensis, Heteroxerus rubricati, Microdyromys koegniswaldi, Microdyromys monspeliensis, Armantomys tricristatus, Megacricetodon collongensis y Democricetodon larteti.

Además, la revisión del material original procedente de Somosaguas-Sur, ha permitido ampliar el listado de especies de micromamíferos presentes en este yacimiento. De esta forma, a las ya conocidas, se añade la presencia de Heteroxerus rubricati, de menor talla que Heteroxerus grivensis, la cual ya se conocía en el yacimiento (Luis \& Hernando, 2000).

Tabla 1.-Abundancias relativas (porcentajes de $\mathrm{m} 1+\mathrm{m} 2+\mathrm{M} 1+\mathrm{M} 2$ ) de las distintas especies en las dos zonas de muestreo y comparación con los resultados del análisis Monte Carlo para 165 molares

\begin{tabular}{|c|c|c|c|c|c|c|}
\hline \multirow[b]{3}{*}{ Especies } & \multirow{3}{*}{$\begin{array}{c}\text { CATA-3 } \\
\% \text { Observado }\end{array}$} & \multicolumn{5}{|c|}{ SOM-S } \\
\hline & & \multirow[b]{2}{*}{$\%$ Observado } & \multicolumn{4}{|c|}{ Análisis Monte Carlo (165 Molares) } \\
\hline & & & $\%$ Medio & Desv. Est. & Rango & $p$ \\
\hline Heteroxerus grivensis & 2,42 & 3,08 & 3,05 & 1,05 & $0,00-6,66$ & 0,124 \\
\hline Heteroxerus rubricati & 1,21 & 1,10 & 1,10 & 0,67 & $0,00-3,03$ & 1,000 \\
\hline Microdyromys koegniswaldi & 9,70 & 7,47 & 7,46 & 1,61 & $1,81-12,72$ & 0,240 \\
\hline Microdyromys monspeliensis & 5,45 & 2,20 & 2,25 & 0,93 & $0,00-5,45$ & $<0,001$ \\
\hline Armantomys tricristatus & 6,67 & 6,59 & 6,64 & 1,56 & $2,42-11,51$ & 1,000 \\
\hline Megacricetodon collongensis & 60,61 & 55,60 & 55,52 & 3,10 & $44,84-64,84$ & 0,126 \\
\hline Democricetodon larteti & 13,94 & 23,96 & 23,97 & 2,59 & $16,36-31,51$ & $<0,001$ \\
\hline
\end{tabular}


Tabla 2.- Resultados de los análisis Monte Carlo y U Mann-Whitney, para las dos muestras estudiadas

\begin{tabular}{|c|c|c|c|c|c|c|c|}
\hline \multirow[b]{3}{*}{ Especies } & \multicolumn{3}{|c|}{ CATA-3 } & \multicolumn{3}{|c|}{ SOM-S } & \multirow{3}{*}{$\begin{array}{c}\text { U Mann } \\
\text { Whitney } \\
p\end{array}$} \\
\hline & \multicolumn{3}{|c|}{ Monte Carlo (100 Molares) } & \multicolumn{3}{|c|}{ Monte Carlo (100 Molares) } & \\
\hline & $\%$ Medio & Desv. Est. & Rango & $\%$ Medio & Desv. Est. & Rango & \\
\hline Heteroxerus grivensis & 2,37 & 0,99 & $0,00-4,00$ & 3,14 & 1,59 & $0,00-10,00$ & $<0,001$ \\
\hline Heteroxerus rubricati & 1,21 & 0,70 & $0,00-2,00$ & 1,07 & 0,92 & $0,00-4,00$ & $<0,001$ \\
\hline Microdyromys koegniswaldi & 9,69 & 1,94 & $2,00-15,00$ & 7,51 & 2,28 & $1,00-15,00$ & $<0,001$ \\
\hline Microdyromys monspeliensis & 5,47 & 1,41 & $1,00-9,00$ & 2,22 & 1,27 & $0,00-7,00$ & $<0,001$ \\
\hline Armantomys tricristatus & 6,66 & 1,63 & $2,00-11,00$ & 6,58 & 2,10 & $1,00-13,00$ & 0,269 \\
\hline Megacricetodon collongensis & 60,63 & 3,10 & $50,00-71,00$ & 55,47 & 4,30 & $41,00-73,00$ & $<0,001$ \\
\hline Democricetodon larteti & 13,96 & 2,19 & $7,00-21,00$ & 24,01 & 3,80 & $12,00-35,00$ & $<0,001$ \\
\hline
\end{tabular}

Con ello, ambas muestras presentan las mismas especies de roedores corroborando una homogeneidad cualitativa. No obstante cuando estudiamos las abundancias relativas de cada especie se aprecian diferencias significativas en las muestras pertenecientes a varias especies.

En el primer análisis de Monte Carlo sobre la muestra de Somosaguas-Sur realizado para 165 molares se obtuvieron diferencias significativas entre esta muestra y la de Cata-3 en las abundancias relativas de dos especies, Microdyromys monspeliensis y Democricetodon larteti (Tabla 1), mientras que el resto de especies no mostraron diferencias a nivel estadístico. En el segundo análisis, en el que ambas muestras fueron sometidas a aleatorizaciones (para 100 molares), los resultados del análisis U de MannWhitney muestran diferencias significativas en todas las especies (Fig. 2), excepto en Armantomys tricristatus (Tabla 2).

\section{Discusión}

Nuestros resultados indican, independientemente de la aproximación utilizada, la existencia de diferencias significativas en las abundancias relativas de varias especies de roedores en las muestras del nivel T1 de Somosaguas (Tabla 1 y 2), tomadas en puntos separados lateralmente menos de $30 \mathrm{~m}$. Esto refutaría la habitualmente asumida homogeneidad lateral en las abundancias de las especies presentes dentro de un mismo nivel fosilífero. Distintas hipótesis pueden ser planteadas para explicar este hecho.

En primer lugar, se podrían plantear dos posibilidades relacionadas con una diferenciación debida a procesos bioestratinómicos (sensu Fernández-López,
2000) asociados con aspectos paleoecológicos previos al enterramiento de los restos. Una primera hipótesis podría implicar la existencia de un sesgo debido a depredación por parte de diferentes especies de aves, con la posterior expulsión espacialmente diferenciada de egagrópilas. Las preferencias alimenticias de distintas rapaces podrían generar un sesgo hacía determinadas especies de roedores, lo que conllevaría una acumulación de restos de estas especies en las zonas próximas a los posaderos de las aves, modificando las abundancias relativas de las distintas especies que se podrían esperar para un ambiente determinado (Terry, 2008, 2010). Además, tenemos que tener en cuenta un factor estacional: las egagrópilas originadas en época seca quedan más tiempo en el posadero y pueden ser desmanteladas antes del transporte, mientras las originadas durante la estación húmeda se transportan inmediatamente y enteras. Esto podría resultar en variaciones laterales a lo largo de un nivel fosilífero, observables como diferencias significativas en las abundancias relativas de distintas especies en zonas próximas. Por otro lado, también se pudo producir un sesgo debido a la posible existencia de microambientes dentro del área de estudio, que condicionasen la abundancia relativa de las distintas especies, las cuales manifestarían una preferencia por un determinado microambiente con condiciones favorables para su desarrollo (Cortés-Calva \& Álvarez Castañeda, 1997; Aragón et al., 2009). No obstante, en ambos casos los procesos de formación del yacimiento de Somosaguas descartan estas posibilidades. Dado que se trata de un yacimiento formado por la acción de abanicos aluviales, que arrastrarían y englobarían los restos de las distintas especies, sería esperable 

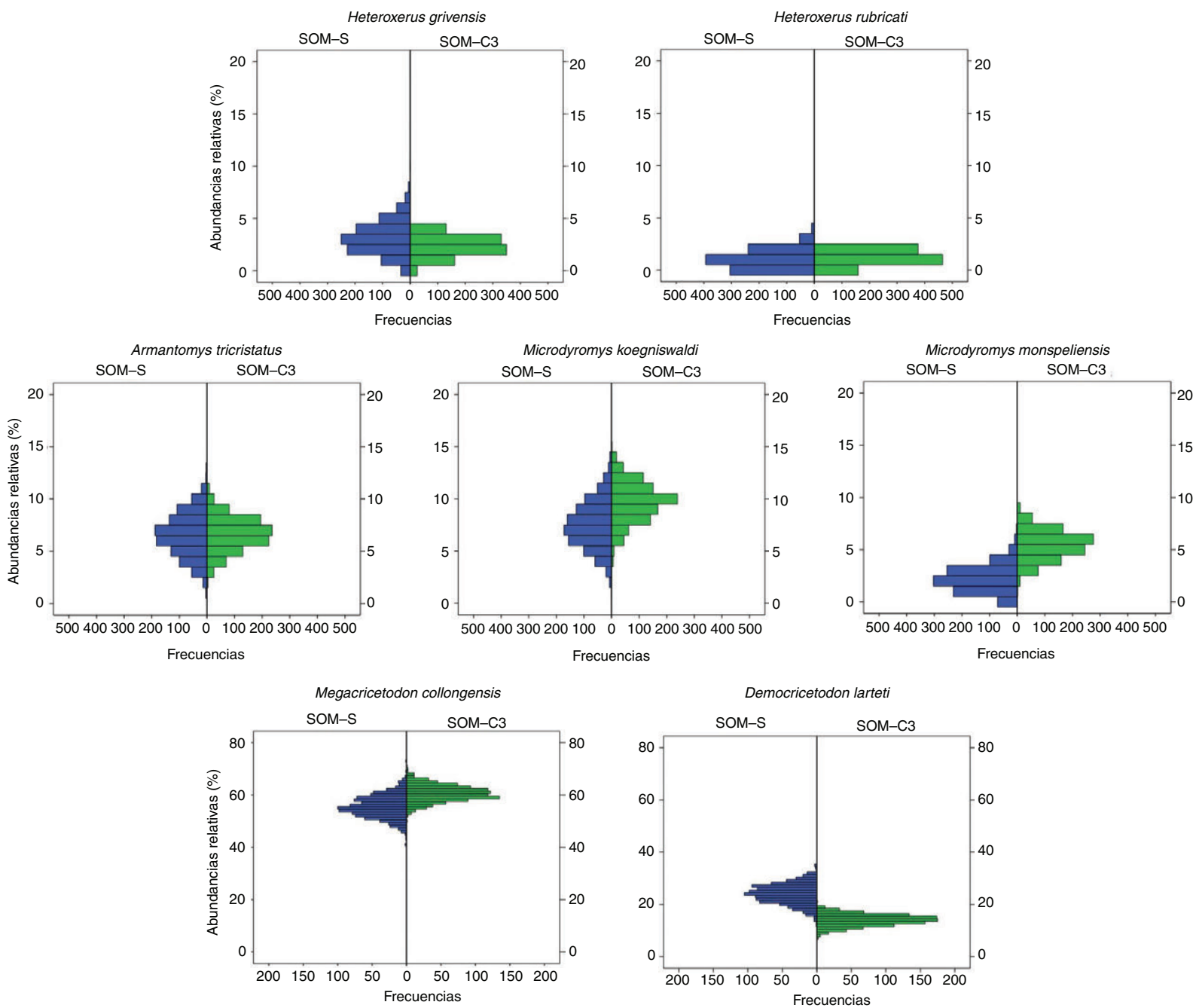

Fig. 2.-Histogramas de abundancias relativas de las especies en Somosaguas-Sur (SOM-S) y CATA-3 (SOM-C3), para las aleatorizaciones realizadas con 100 molares. El eje de ordenadas representa las abundancias relativas (\% porcentajes de $\mathrm{m} 1+\mathrm{m} 2+\mathrm{M} 1+\mathrm{M} 2)$ de las especies estudiadas; el eje de abscisas representa las frecuencias (número de aleatorizaciones) obtenidas en el análisis Monte Carlo para cada abundancia relativa. Para mayor claridad todas las tablas están a la misma escala, excepto las pertenecientes a Megacricetodon collongensis y Democricetodon larteti, debido a la mayor abundancia de estas especies.

una homogenización de estas supuestas diferencias iniciales, ya fueran debidas a las preferencias de los depredadores, a la acumulación espacialmente diferenciada de los restos de distintas especies o a la existencia de microambientes que modificaran las abundancias relativas de las paleocomunidades originales. Del mismo modo, las áreas de campeo de los depredadores implicados generalmente no se restringe a un microambiente determinado, generando una acumulación de restos que incluye una mezcla de especies representantes de distintos microambientes. Por tanto, debemos buscar posibles causas de diferenciación que no se vean afectadas o incluso que incluyan los procesos de formación del yacimiento.

En ocasiones se ha observado una relación entre el tamaño corporal y la abundancia de las especies en los yacimientos (Palmquist et al., 1992), lo cual podría explicar las diferencias observadas en las abundancias de las especies en distintos puntos de muestreo del tramo T1 de Somosaguas. A su vez, esto podría estar relacionado con los procesos sedimentarios propios de abanicos aluviales, en los cuales la energía deposicional varía a lo largo de su extensión generando un arrastre diferencial de restos de distinto tamaño, depositándolos de manera sesgada conforme 
se va perdiendo energía (Bermúdez \& García, 1990). Este proceso implicaría una tendencia hacia la sobrerrepresentación de las especies de mayor o menor tamaño con respecto al resto en una de las dos muestras. En nuestra primera aproximación analítica, basada en la aleatorización únicamente de la muestra procedente de Somosaguas-Sur, observamos que las dos especies que muestran diferencias significativas entre Somosaguas-Sur y la Cata-3, Microdyromys monspeliensis y Democricetodon larteti (Tabla 1), se encuentran situadas en ambos extremos del gradiente de tamaño dental. Si analizamos las diferencias en las abundancias relativas entre las dos aleatorizaciones realizadas en nuestra segunda aproximación analítica (Tabla 2) vemos que también existe una tendencia hacia un incremento relativo entre SomosaguasSur y la Cata-3 de la abundancia de las especies de menor talla (Heteroxerus rubricati, Microdyromys koegniswaldi, Microdyromys monspeliensis y Megacricetodon collongensis) frente a las de mayor tamaño (Heteroxerus grivensis y Democricetodon larteti). Parece ser que incluir un factor de variabilidad, por medio de la comparación de aleatorizaciones en ambas muestras, incrementa la sensibilidad estadística del análisis con respecto a la aproximación basada directamente en los datos observados en la Cata-3. Únicamente Armantomys tricristatus, la especie más grande de la asociación, no ha mostrado diferencias en su abundancia relativa entre ambos puntos de muestreo en ninguno de los dos análisis realizados. Estos resultados parecen sugerir la existencia de diferencias en el comportamiento como partículas sedimentarias de los restos dentales de las diferentes especies, en relación con sus distintos tamaños y con una diferencia en la energía entre partes más o menos distales del abanico aluvial en el que se formó la acumulación de restos del tramo T1.

Por otro lado, el tramo T1 de Somosaguas constituye uno de los yacimientos fluvio-lacustres con mayor densidad de restos de microfósiles de todo el Cenozoico ibérico (López-Martínez et al., 2000; y el presente trabajo). Ambas muestras recogidas se plantean como representativas desde un punto de vista faunístico dado que, como sugieren Weerd \& Daams (1978) incluyen más de un centenar de primeros y segundos molares. No obstante, la extrema densidad de fósiles que encontramos en Somosaguas podría generar un problema de representatividad de la muestra desde el punto de vista sedimentológico, ya que sólo fue necesario coger una muestra de sedimento muy pequeña (menos de $100 \mathrm{Kg}$ ) en cada uno de los puntos de muestreo para alcanzar ese elevado número de fósiles, cuando en otros yacimientos no kársticos de edades similares es necesaria la recogida de varias toneladas de sedimento para alcanzar niveles similares de riqueza fosilífera (Daams \& Freudenthal, 1988). Muestras de sedimento de mayor calibre que las recogidas hasta la fecha podrían resultar en una homogeneización faunística de las mismas.

Una última hipótesis a plantear radica en la posibilidad de que el tramo T1, con una potencia de unos $57 \mathrm{~cm}$ (Díez-Canseco et al., 2012), corresponda a varios eventos sedimentarios sucesivos no detectables estratigráficamente. El tipo de muestreo utilizado en el tramo T1 para recuperar los fósiles de microvertebrados (lavado-tamizado), hace que la ubicación de los mismos dentro del estrato sea imposible. Esto no ocurre con los métodos de excavación tradicional, llevados a cabo en la extracción de macrofauna como es el caso de Somosaguas Norte dentro del tramo T3 (López-Martínez et al., 2000). En este estrato debido a los datos procedentes de la geolocalización de todos los restos obtenidos en las campañas de excavación, se ha podido llevar a cabo una identificación precisa de capas dentro de un nivel estratigráfico aparentemente homogéneo que corresponden a abanicos aluviales sucesivos (Elez, 2005; Hernández Fernández et al., 2006; Martín, 2015), coincidiendo con un proceso de enfriamiento y aridificación del macroclima regional (Domingo et al., 2009). No obstante, estudios anteriores ya han observado diferencias en la composición mineralógica del mismo (Fesharaki, 2005; Fesharaki et al., 2007; Díez-Canseco, 2011) lo cual apunta a posible presencia de varios abanicos aluviales superpuestos. Por tanto, teniendo en cuenta la reducida cantidad de sedimento necesaria para el muestreo faunístico de este tramo, podría darse una cierta mezcla temporal (no significativa sensu Kowalewski, 1996) diferencial entre la muestra recogida en Somosaguas-Sur y la Cata-3, que se relacionaría con potenciales diferencias en la distribución lateral de distintos abanicos sucesivos, afectando de manera diferenciada a cada punto de muestreo. Además, el Aragoniense Medio es una época de marcada estacionalidad con 
precipitaciones escasas y probablemente impredecibles, lo que pudo dar lugar a sequías de duración variable (Domingo et al., 2009; García Yelo et al., 2014; Martín, 2015). La escasez de agua puede llegar a ser el factor limitante con mayor efecto sobre la dinámica poblacional de las comunidades de roedores (Morgan Ernest et al., 2000; Stapp, 2010; Heisler et al., 2014; Shenbrot, 2014). Las abundancias relativas de las diferentes especies de roedores de las zonas áridas del Desierto de Chihuahua (Nuevo México y Arizona) sufren variaciones anuales significativas asociadas a la disponibilidad hídrica (Morgan Ernest et al., 2000). Teniendo en cuenta el cambio de escala temporal, resulta plausible una diferenciación significativa en las abundancias relativas especificas en muestras en el clima semiárido dominante en el área de Madrid durante el Aragoniense Medio que podrían pertenecer a eventos de sedimentación diferenciados. Esta situación de clima árido con disponibilidad hídrica variable que afectase de manera significativa variando las abundancias relativas de las distintas especies en unos pocos miles de años, sumado al hecho de una hipotética formación del nivel T1 por medio de varios abanicos aluviales sucesivos, daría una explicación posible a la diferenciación observada en el presente estudio en las abundancias de las especies de roedores muestreadas en dos puntos del yacimiento de Somosaguas.

Estas tres últimas hipótesis planteadas, ya sea de manera independiente o por combinación de los procesos asociados a varias de ellas, podrían explicar las diferencias observadas en las abundancias relativas de las diferentes especies registradas en la Cata-3 con respecto a Somosaguas-Sur. Para dilucidar la exclusividad de alguna de las hipótesis o la complementariedad de varias de ellas, resultarán necesarios futuros estudios que aborden muestreos en yacimientos con diferentes características estructurales y de densidad de restos.

\section{Conclusiones}

Este estudio deja patente la existencia de diferencias estadísticamente significativas en las abundancias relativas en las dos muestras estudiadas del tramo T1 del yacimiento de Somosaguas, mostrando una variación lateral no detectada previamente en un mismo nivel fosilífero. La explicación para estos resultados podría relacionarse con 1) la existencia de un diferente comportamiento sedimentario de las piezas dentales correspondientes a las distintas especies debido a diferencias en su tamaño; 2) el reducido tamaño de la muestra de sedimento en combinación con la elevada densidad de restos fósiles en el tramo T1 de Somosaguas; y 3) una mezcla temporal diferencial relacionada con la presencia de varios abanicos aluviales sucesivos, sumado a posibles cambios en las dinámicas poblacionales de las comunidades de roedores durante el Aragoniense Medio en el área de Madrid, favorecidas por el clima de precipitaciones impredecibles que conllevaba épocas de sequias prolongadas.

La detección de esta heterogeneidad lateral en las abundancias relativas específicas en un mismo nivel fosilífero nos muestra la necesidad de ser cautelosos al hacer interpretaciones paleocológicas basadas en abundancias, especialmente en los yacimientos más ricos (densos).

\section{AGRADECIMIENTOS}

Queremos agradecer a Nieves López Martínez y los miembros del Proyecto Somosaguas de Paleontología su inestimable colaboración en la excavación de la Cata-3 así como en la posterior recogida y lavado de las muestras de sedimento del tramo T1. Así mismo, agradecemos los valiosos comentarios de Davinia Díez-Canseco, Israel García Paredes, Omid Fesharaki, Adriana Oliver y Verónica Hernández Ballarín que nos han ayudado a mejorar la discusión de los resultados. A su vez, queremos agradecer a Matthijs Freudenthal y a un revisor anónimo por sus acertados comentarios, los cuales nos han servido para mejorar el manuscrito original. Este trabajo es una contribución del equipo de Paleoclimatología, Macroecología y Macroevolución de Vertebrados (www.pmmv.com.es) de la Universidad Complutense de Madrid como parte del grupo de investigación UCM 910607 sobre Evolución de Mamíferos y Paleoambientes Continentales Cenozoicos.

\section{Referencias}

Aguilar, J.P. (1977). Les gisements continentaux de Plaissan et de la Nouvelle Faculte de Medecine (Herault) leur position stratigraphique. Geobios, 10 (1): 81-101. http://dx.doi.org/10.1016/S0016-6995(77)80055-7

Anemone, R.L.; Dawson, M.R. \& Beard, K.C. (2012). The Early Eocene Rodent Tuscahomys (Cylindrodontidae) from the Great Divide Basin, Wyoming: Phylogeny, Biogeography, and Paleoecology. Annals of Carnegie Museum, 80 (3): 187-205. http://dx.doi. org/10.2992/007.080.0302 
Aragón, E.E.; Garza, A. \& Cervantes, F.A. (2009). Estructura y organización de los ensambles de roedores de un bosque de la Sierra Madre Occidental, Durango, México. Revista Chilena de Historia Natural, 82 (4): 523-542. http://dx.doi.org/10.4067/ S0716-078X2009000400007

Bermúdez, F.L. \& García, C.C. (1990). Características granulométricas de los depósitos aluviales en el Campo de Cartagena. Cuadernos de investigación geográfica, 16: 31-54.

Casanovas-Vilar, I. \& Agusti, J. (2007). Ecogeographical stability and climate forcing in the Late Miocene (Vallesian) rodent record of Spain. Palaeogeography, Palaeoclimatology, Palaeoecology, 248 (1-2): 169189. http://dx.doi.org/10.1016/j.palaeo.2006.12.002

Cortés-Calva, P. \& Álvarez Castañeda, S.T. (1997). Diversidad de roedores en la Bahia de La Paz, Baja California Sur. In: Urbán, R.J. \& Ramírez, M. (Eds.). La bahía de La Paz: investigación y conservación. Universidad Autónoma Baja California Sur, Centro Interdisciplinario de Ciencias del Mar, Institute of Oceanography, p. 265-272.

Crusafont, M.; Villalta, J.F. \& Truyols, Y. (1955). El Burdigaliense continental de la cuenca del Vallès-Penedès. Barcelona, CSIC, 273 p.

Cuenca-Bescós, G.; Straus, L.G.; Gónzalez Morales, M.R. \& García Pimienta, J.C. (2009). Paleoclima y paisaje del final del Cuaternario en Cantabria: los pequeños mamíferos de la Cueva del Mirón (Ramales de la victoria). Revista Española de Paleontología, 23 (1): 91-126.

Cuevas-González, J. (2005). Estado actual de los conocimientos paleontológicos y estratigráficos de los yacimientos aragonienses de Somosaguas, (Pozuelo de Alarcón, Madrid). Coloquios de Paleontología, 55: 103-123.

Daams, R. \& Meulen, A.J. van der (1984). Paleoenviromental and paleoclimatic interpretation of micromammal faunal succesions in the Upper Oligocene and Miocene of North Central Spain. Paléobiologie Continentale, 14: 241-257.

Daams, R. \& Freudenthal, M. (1988). Synopsis of the Dutch-Spanish collaboration program in the Aragonian type area, 1975-1986. Scripta Geologica, 1: 3-18.

Daams, R.; Meulen, A.J. van der; Peláez-Campomanes, P. \& Álvarez-Sierra, M.A. (1999). Trends in rodent assemblages from the Aragonian (early-middle Miocene) of the Calatayud-Daroca Basin, Aragon, Spain. In: Agustí, J; Rook, L. \& Andrews, P. (Eds.). Hominoid evolution and climatic change in Europe. Cambridge University Press, Cambridge, Volume 1, p. 127-139.

Dam, J.A. van (1997). The small mammals from the upper Miocene of the Teruel-Alfambra region (Spain): paleobiology and paleoclimatic reconstructions. Geologica Ultraiectina, 156: 1-204. http://dspace. library.uu.nl/handle/1874/268355
De Bruijn, H. (1966). Some new Miocene Gliridae (Rodentia, Mammalia) from the Calatayud area (prov. Zaragoza, Spain). Proceedings of the Koninklijke Nederlandse Akademie Van Wetenschappen, Series B, 69 (1): 58-71.

Díez-Canseco, D. (2011). Estudio sedimentológico del tramo T2 de los yacimientos paleontológicos de Somosaguas. Trabajo Fin de Máster en Ciencias Geológicas, Universidad Complutense de Madrid, 50 p.

Díez-Canseco, D.; López-Martínez, N.; Díaz-Molina, M. \& Benito, M.I. (2012). Stream mouth deposits in the palaeontological site of Somosaguas, Middle Miocene, Madrid Basin. Spanish Journal of Palaeontology, 27 (2): 93-104. http://eprints.ucm.es/29845/

Domingo, L.; Cuevas-González, J.; Grimes, S.T.; Hernández Fernández, M. \& López-Martínez, N. (2009). Multiproxy reconstruction of the palaeoclimate and palaeoenvironment of the Middle Miocene Somosaguas site (Madrid, Spain) using herbivore dental enamel. Palaeogeography, Palaeoclimatology, Palaeoecology, 272 (1-2): 53-68. http://dx.doi. org/10.1016/j.palaeo.2008.11.006

Elez, J. (2005). Aplicación GIS 3D a los yacimientos paleontológicos de Somosaguas. Trabajo de Investigación Tutelado para la obtención del Diploma de Estudios Avanzados. Universidad Complutense de Madrid, 39 p.

Fernández García, M. (2014). Paleoecología y biocronología mediante el estudio de los roedores del Pleistoceno Superior-Holoceno de la cueva del Toll (Moià, Cataluña, NE de la península Ibérica). Treballs del Museu de Geologia de Barcelona, 20 (1): 73-97.

Fernández-López, S.R. (2000). Temas de Tafonomía. Departamento de Paleontología, Universidad Complutense de Madrid, $167 \mathrm{p}$.

Fesharaki, O. (2005). Mineralogía y Sedimentología del yacimiento Paleontológico de Somosaguas (Mioceno, Cuenca de Madrid). Trabajo de Investigación Tutelado para la obtención del Diploma de Estudios Avanzados. Universidad Complutense de Madrid, $147 \mathrm{pp}$.

Fesharaki, O.; García-Romero, E.; Cuevas-González, J. \& López-Martínez, N. (2007). Clay mineral genesis and chemical evolution in the Miocene sediments of Somosaguas, Madrid Basin, Spain. Clay Minerals, 42 (2): 187-201. http://dx.doi.org/10.1180/ claymin.2007.042.2.05

Forsyth Major, C.J. (1893). On some Miocene squirrels, with remarks on the dentition and classification of Sciuridae. Proceedings of the Zoological Society of London, 1893: 179-215. http://biodiversitylibrary. org/page/30981178

Freudenthal, M. (1963). Entwicklungsstufen der miozänen Cricetodontinae (Mammalia, Rodentia) Mittelspaniens und ihre stratigrafische Bedeutung. Beaufortia 10: 51-157. http://www.repository.naturalis.nl/document/548592 
García Yelo, B.A.; Gómez Cano, A.R.; Cantalapiedra, J.L.; Alcalde, G.M.; Sanisidro, O.; Oliver, A.; Hernández-Ballarín, V.; López-Guerrero, P.; Fraile, S. \& Hernández Fernández, M. (2014). Palaeoenvironmental analysis of the Aragonian (middle Miocene) mammalian faunas from the Madrid Basin based on body-size structure. Journal of Iberian Geology, 40 (1): 129-140. http://dx.doi.org/10.5209/ rev_JIGE.2014.v40.n1.44092

Gómez Cano, A.R.; Cantalapiedra, J.L.; Álvarez-Sierra, M.Á. \& Hernández Fernández, M. (2014). A macroecological glance at the structure of late Miocene rodent assemblages from Southwest Europe. Scientific Reports, 4: 6557. http://dx.doi.or/10.1038/ srep06557

Hammer, O.; Harper, D.A.T. \& Ryan P.D. (2001). PAST: Paleontological Statistics Software Package for Education and Data Analysis. Paleontología Electrónica, $4(1): 4$.

Heisler, L.M.; Somers, C.M. \& Poulin, R.G. (2014). Rodent populations on the northern Great Plains respond to weather variation at a landscape scale. Journal of Mammalogy, 95 (1): 82-90. http://dx.doi. org/10.1644/13-MAMM-A-115.1

Hernández-Ballarín, V.; Oliver, A. \& Peláez-Campomanes, P. (2011). Revisión de las asociaciones de mamíferos del tránsito Aragoniense medio y superior de la cuenca de Madrid. In: Pérez-García, A.; Gascó, F.; Gasulla, J.M. \& Escaso, F. (Eds.). Viajando a Mundos Pretéritos. Ajuntament de Morella, 173-182.

Hernández Fernández, M. (2006). Rodent faunas as indicators of climatic change in Europe during the last 125,000 years. Quaternary Research, 65 (2): 308323. http://dx.doi.org/10.1016/j.yqres.2005.08.022

Hernández Fernández, M.; Cárdaba, J.A.; Cuevas-González, J.; Fesharaki, O.; Salesa, M.J.; Corrales, B.; Domingo, L.; Élez, J.; López Guerrero, P.; Sala-Burgos, N. \& López Martínez, N. (2006). Los yacimientos de vertebrados del Mioceno medio de Somosaguas (Pozuelo de Alarcón, Madrid): implicaciones paleoambientales y paleoclimáticas. Estudios Geológicos, 62 (1): 263-294. http://dx.doi. org/10.3989/egeol.0662126

Hernández Fernández, M.; Álvarez-Sierra, M.Á. \& Peláez-Campomanes, P. (2007). Bioclimatic analysis of rodent palaeofaunas reveals severe climatic changes in Southwestern Europe during the Plio-Pleistocene. Palaeogeography, Palaeoclimatology, Palaeoecology, 251 (3-4): 500-526. http://dx. doi.org/10.1016/j.palaeo.2007.04.015

Kasuya, E. (2001). Mann-Whitney U test when variances are unequal. Animal Behaviour, 61 (6): 1247-1249. http://dx.doi.org/10.1006/anbe.2001.1691

Kowalewski, M. (1996). Time-averaging, overcompleteness, and the geological record. The Journal of Geology, 104 (3): 317-326. http://dx.doi. org/10.1086/629827
López-García, J.M.; Agustí, J. \& Aouraghe, H. (2013). The small mammals from the Holocene site of Guenfouda (Jerada, Eastern Morocco): chronological and paleoecological implications. Historical Biology, 25 (1): 51-57. http://dx.doi.org/10.1080/08912963.2012. 688198

López-Martínez, N.; Sesé Benito, C. \& Sanz García, J.L. (1977). La microfauna (rodentia, insectívora, lagomorpha y reptilia) de las fisuras del Mioceno medio de Escobosa de Calatañazor (Soria, España). Acta Geológica Hispánica, 12 (1): 60-68.

López-Martínez, N.; Élez, J.; Hernando, J.M.; Luis Cavia, A.; Mazo, A.; Mínguez Gandú, D.; Morales, J.; Polonio, I.; Salesa, M.J. \& Sánchez, I.M. (2000). Los fósiles de vertebrados de Somosaguas (Pozuelo, Madrid). Coloquios de Paleontología, 51: 71-85. http://hdl.handle.net/10261/8565

Luis Cavia, A. (2002). Aplicación de la rarefacción al yacimiento de microvertebrados de Somosaguas (Pozuelo de Alarcón, España). Tesis de Licenciatura, Universidad Complutense de Madrid, 66 pp.

Luis, A. \& Hernando, J.M. (2000). Los microvertebrados del Mioceno Medio de Somosaguas Sur (Pozuelo de Alarcón, Madrid, España). Coloquios de Paleontología, 51: 87-136.

Lyman, R. (2014). Paleoenvironmental implications of two relative indicator rodent taxa during the Pleistocene to Holocene transition in south-eastern Washington state, USA. Journal of Quaternary Science, 29 (7): 691-697. http://dx.doi.org/10.1002/jqs.2737

Manly, B.F.J. (1997). Randomization, Bootstrap and Monte Carlo Methods in Biology. Chapman \& Hall, London, $480 \mathrm{pp}$.

Martín, D.M. (2015). New taphonomic inferences on the Middle Miocene vertebrate site of Somosaguas (Pozuelo de Alarcón, Madrid). Trabajo Fin de Master, Facultad de Ciencias, Universidad Complutense de Madrid, 46 pp.

Mein, P. (1958). Les mammifères de la faune sidérolithique du Vieux-Collonges. Nouvelles Archives du Muséum d'Histoire Naturelle de Lyon, 5: 1-122.

Menéndez, I.; Gómez Cano, A.R. \& Hernández Fernández, M. (2015). Patrón de mortandad de Megacricetodon collongensis (MEIN, 1958) en Somosaguas Sur (Mioceno medio, Pozuelo de Alarcón, Madrid). In: Domingo, L.; Domingo, M.S.; Fesharaki, O.; García Yelo, B.A.; Gómez Cano, A.R.; Hernández-Ballarín, V.; Hontecillas, D.; Cantalapiedra, J.L.; López Guerrero, P.; Oliver, A.; Pelegrín, J.; Pérez de los Ríos, M.; Ríos, M.; Sanisidro, O. \& Valenciano, A. (Eds.). Current trends in Paleontology and Evolution: Conference proceedings XIII Meeting of Early-Stage Researchers in Paleontology, 191-192.

Meulen, A.J. van der \& Daams, R. (1992). Evolution of Early-Middle Miocene rodent faunas in relation to longterm palaeoenvironmental changes. Palaeogeography, 
Palaeoclimatology, Palaeoecology, 93 (3-4): 227-253. http://dx.doi.org/10.1016/0031-0182(92)90099-Q

Meulen, A.J. van der; Peláez-Campomanes, P. \& Daams, R. (2003). Revision of medium-sized Cricetidae from the Miocene of the Daroca-Villafeliche area in the Calatayud-Teruel basin (Zaragoza, Spain). Coloquios de Paleontología, 1: 385-441. http://eprints. ucm.es/10898/

Mínguez Gandú, D. (2000). Marco estratigráfico y sedimentológico de los yacimientos paleontológicos miocenos de Somosaguas (Madrid, España). Coloquios de Paleontología, 51: 183-196.

Morgan Ernest, S.K.; Brown, J.H. \& Parmenter, R.R. (2000). Rodents, plants and precipitation: spatial and temporal dynamics of consumer and resources. Oikos, 88 (3): 470-482. http://dx.doi. org/10.1034/j.1600-0706.2000.880302.x

Oliver, A. (2015). Evolución del género Megacricetodon del Aragoniense y Vallesiense (Mioceno) de la Península Ibérica. Tesis doctoral, Universidad Complutense de Madrid, 316 p. http://eprints.ucm.es/30150/

Palmquist, P; Gibert, J. \& Martínez, B. (1992). Sobre la relación tamaño/abundancia en los macromamíferos de Venta Micena y sus implicaciones tafonómicas. Revista Española de Paleontología, 7 (2): 174-180.

Perales, R.; Serrano, H.; García Yelo, B.A. \& Hernández Fernández, M. (2009). Inferencias paleoambientales del Mioceno medio de Somosaguas (Pozuelo de Alarcón, Madrid) basadas en la estructura de tamaños corporales de su fauna de mamíferos. Paleolusitana, 1: 317-325. http://eprints.sim.ucm.es/15597/

Polonio, I. \& López Martínez, N. (2000). Análisis tafonómico de los yacimientos de Somosaguas (Mioceno medio, Madrid). Coloquios de Paleontología, 51: $235-265$.

Rodrigues, H.G.; Marivaux, L. \& Vianey-Liaud, M. (2012). Expansion of open landscapes in Northern China during the Oligocene induced by dramatic climate changes: Paleoecological evidence. Palaeogeography, Palaeoclimatology, Palaeoecology, 358-360: 62-71. http://dx.doi.org/10.1016/j.palaeo.2012.07.025

Rodríguez Fernández, L.R.; López Olmedo, F.; Oliveira, J.T.; Medialdea, T.; Terrinha, P.; Matas, J.; MartínSerrano, A.; Martín Parra, L.M.; Rubio, F.; Marín, C.; Montes, M. \& Nozal, F. (2016). Mapa Geológico de la Península Ibérica, Baleares y Canarias a escala 1:1.000.000, edición 2015. Instituto geológico y minero de España (IGME) \& Laboratorio
Nacional de Energía y Geología de Portugal (LNEG), Madrid.

Sainz de los Terreros, J.Y. \& Castanedo, A.G. (2011). Análisis de los procesos tafonómicos de Cueva Morín. Primeros resultados de un estudio necesario. Zephyrvs, 67: 69-90.

Schaub, S. (1925). Die Hamsterartige Nagetiere des Tertiärs und ihre lebenden Verwandten. Mémoires de la Société Paléontologique Suisse, 45: 1-114.

Sesé, C. (1991). Interpretación paleoclimática de las faunas de micromamíferos del Mioceno, Plioceno y Pleistoceno de la cuenca de Guadix-Baza (Granada, España). Estudios Geológicos, 47 (1-2): 73-83. http://dx.doi.org/10.3989/egeol.91471-2409

Sesé, C. (2011). Micromamíferos (Erinaceomorfos y Roedores) del final del Pleistoceno Superior y primera parte del Holoceno de Cova Fosca (Alto Maestrazgo, Castellón): Reconstrucción paleoambiental del entorno del yacimiento. Archaeofauna, 20: 119-137. http://hdl.handle.net/10261/45324

Shenbrot, G. (2014). Population and community dynamics and habitat selection of rodents in complex desert landscapes. Mammalia, 78 (1): 1-10. http:// dx.doi.org/10.1515/mammalia-2013-0066

Stapp, P. (2010). Long-term studies of small mammal communities in arid and semiarid environments. Journal of Mammalogy, 91 (4): 773-775. http://dx. doi.org/10.1644/10-MAMM-S-154.1

Terry, R. (2008). Modeling the effects of predation, prey cycling, and time averaging on relative abundance in raptor-generated small mammal death assemblages. Palaios, 23 (6): 402-410. http://dx.doi.org/10.2110/ palo.2007.p07-071r

Terry, R. (2010). On raptors and rodents: testing the ecological fidelity and spatiotemporal resolution of cave death assemblages. Paleobiology, 36 (1): 137-160. http://dx.doi.org/10.1666/0094-8373-36.1.137

Voglino, D. \& Pardiñas, U.F. (2005). Roedores sigmodontinos (Mammalia: Rodentia: Cricetidae) y otros micromamíferos pleistocénicos del norte de la provincia de Buenos Aires (Argentina): reconstrucción paleoambiental para el Ensenadense cuspidal. Ameghiniana, 42 (1): 143-158.

Weerd, A. van de \& Daams, R. (1978). Quantitative composition of rodent faunas in the Spanish Neogene and paleoecological implications. Proceedings of the Koninklijke Nederlandse Akademie van Wetenschappen, B, 81, 4: 448-473. 